\title{
Shifted Landau ladders and low field magneto-oscillations in high-mobility GaAs 2D hole systems
}

\author{
Po Zhang, ${ }^{1}$ Jianli Wang, ${ }^{1}$ Chi Zhang, ${ }^{1,2}$ Rui-Rui Du, ${ }^{1,2,3}$ L. N. Pfeiffer, ${ }^{4}$ and K. W. West ${ }^{4}$ \\ ${ }^{1}$ International Center for Quantum Materials, Peking University, Beijing 100871, China \\ ${ }^{2}$ Collaborative Innovation Center of Quantum Matter, Beijing 100871, China \\ ${ }^{3}$ Department of Physics and Astronomy, Rice University, Houston, Texas 77251-1892, USA \\ ${ }^{4}$ Department of Electrical Engineering, Princeton University, Princeton, New Jersey 08544, USA
}

(Received 18 December 2016; accepted 1 March 2017; published online 16 March 2017)

\begin{abstract}
We present well-developed low-field magneto-resistance oscillations originating from zero-field spin splitting (ZFSS) of heavy holes in high mobility GaAs/AlGaAs quantum wells. This low field oscillation is $1 / B$-periodic and emerges before the onset of Shubnikov-de Haas oscillations. The effect can be explained by resonant scattering between two Landau ladders shifted by the ZFSS gap, which in turn can be measured by comparing with the hole cyclotron energy. A front gate is fabricated to tune the ZFSS and hence the oscillation period. Published by AIP Publishing.

[http://dx.doi.org/10.1063/1.4978673]
\end{abstract}

Spin orbital coupling (SOC) in semiconductors is very attractive in both fundamental research ${ }^{1}$ and electronic applications. ${ }^{2}$ In GaAs two-dimensinal hole systems (2DHS), the uppermost valence band is the heavy hole band. The spin degeneracy of heavy holes is killed by SOC arising from the inverse asymmetry of the Zinc-Blende structure and the confinement potential in the growth direction, resulting in a zero field spin splitting (ZFSS). In previous transport measurements, ZFSS is usually analyzed through the beating patterns of Shubnikov-de Haas ( $\mathrm{SdH}$ ) oscillations from two different spin subbands. ${ }^{3-10}$ The carrier density of each spin subband can be obtained from the FFT spectrum of magnetoresistance. The splitting gap can then be calculated using the densities and theoretically predicted energy dispersion.

Thanks to the improved quality in the 2DHS wafer growth; we report our study on another type of magnetoresistance oscillations caused by ZFSS, which appear at lower magnetic fields (B) where $\mathrm{SdH}$ remains unresolved. We propose that this low field oscillation is a consequence of resonant scattering between two spin subbands, which is similar to the case of electric subbands or called confinement subbands. ${ }^{11,12}$ The scattering is enhanced each time when the gap between two subbands $\Delta$ equals to an integral multiple of the cyclotron energy $\hbar \omega_{c}$ (Fig. 1(c)), i.e., 1/B $\propto \Delta / \hbar \omega_{c}=1,2,3 \ldots$, which leads to an $1 / \mathrm{B}$-periodic term in low field magneto-resistances. Although the phenomenon has already appeared in high mobility hole systems ${ }^{3}$ or when the ZFSS is enhanced by an external electric field, ${ }^{6}$ few works have focused on this effect. In our study, the $1 / B$-periodic term can be used to determine the gap of ZFSS and can be tuned by a front gate.

We prepare 3 samples from 2 wafers in our study. Each wafer contains a $20 \mathrm{~nm}(001) \mathrm{GaAs} / \mathrm{AlGaAs}$ quantum well with double-sided symmetrical carbon $\delta$-doping layers grown by MBE. Samples A and B are in van der Pauw geometries, and sample $\mathrm{C}$ has a Hall bar mesa with the $\mathrm{Ti} / \mathrm{Au}$ front gate. A dielectric layer of the gate device is fabricated by PMMA. Samples B and C are cleaved from the same wafer. The details of these samples are listed in
Table I. Our low field experiments are carried out in a cryogen-free dilution refrigerator (DR) with a base temperature of about $40 \mathrm{mK}$. The temperature-dependent $\mathrm{SdH}$ oscillations for effective mass estimation are taken in a $\mathrm{He} 3$ system with a base temperature of about $270 \mathrm{mK}$. Fig. 1(a) gives an overview of magneto-transport data in sample A. The features of fractional quantum Hall states (FQHS) around filling factor $3 / 2$ and $5 / 2$ are pronounced, exhibiting the high quality of our wafers.

The low field oscillations in samples A and B are shown in Fig. 1(b). The oscillations cannot be explained as the beating pattern of SdHs from two different spin subbands ${ }^{3-10}$ since the $\mathrm{SdHs}$ are still unresolved at such low magnetic fields. Similar phenomena may be observed due to the resonance with microwave, phonons, or unintentional periodic

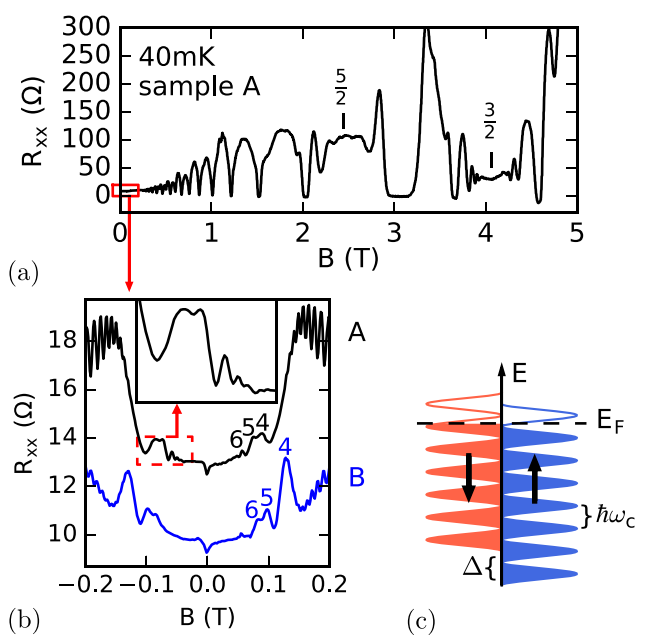

FIG. 1. (a) An overview of magneto-resistance in sample A. Welldeveloped minima of FQHS around $\nu=5 / 2$ and 3/2 show the high quality of wafers. (b) ZFSS induced low field oscillations in samples A and B. The data in panels (a) and (b) are from different traces. The low field oscillations arise when the $\mathrm{SdH}$ oscillations are still unresolved. The inset in panel (b) shows the zoomed-in features in dashed rectangle. The local maximum of $R_{x x}$ occurs when the spin-splitting gap is an integer multiple (denoted by "index," noted at the peaks) of cyclotron energy. A diagram depicting zero filed spin splitting and the Landau ladders is shown in panel (c). 
TABLE I. Information on samples used in this work. Samples A and B are of van der Pauw configurations. Sample $\mathrm{C}$ is a Hall bar device. B and C are cleaved from the same wafer. All 2DHSs are in $20 \mathrm{~nm}$ wide, C-doped, (001) GaAs/AlGaAs quantum wells, with a symmetrical (double-sided) doping. Sample $\mathrm{C}$ has a Ti/Au front gate with a PMMA dielectric layer. $\Delta$ indicates the gap of zero field spin splitting in heavy-hole band. $B_{i=1}$ indicates the magnetic field where $\hbar \omega_{\mathrm{c}} / \Delta=1$.

\begin{tabular}{lccc}
\hline \hline & $\mathrm{A}$ & $\mathrm{B}$ & $\mathrm{C}^{\mathrm{a}}$ \\
\hline Density $\left(10^{11} \mathrm{~cm}^{-2}\right)$ & 1.41 & 1.91 & 1.85 \\
Mobility $\left(10^{6} \mathrm{~cm}^{2} / \mathrm{Vs}\right)^{\mathrm{b}}$ & 1.99 & 1.86 & 1.46 \\
Effective mass $\left(m_{e}\right)$ & 0.54 & 0.44 & - \\
$\Delta(\mathrm{meV})$ & 0.07 & 0.13 & $0.12^{\mathrm{c}}$ \\
$B_{i=1}(\mathrm{~T})$ & 0.34 & 0.50 & 0.45 \\
\hline \hline
\end{tabular}

a The parameters are estimated with the gate grounded.

${ }^{\mathrm{b}}$ The mobility is measured in a cryogen-free DR with a base temperature of $\sim 40 \mathrm{mK}$.

${ }^{\mathrm{c}}$ Assuming the effective mass is the same as sample B.

defects generated during the growth (geometric resonance). Microwave and phonons can be ruled out because there is no intentional microwave irradiation during our experiments nor does the low field oscillation enhance when the temperature increases (more phonons at higher temperature). If this phenomenon is due to the defects from the substrate, the period of defects should be about $300 \mathrm{~nm}$ and very uniform, which is unreasonable. Furthermore, since the phenomenon is common in high mobility hole samples, it should be easier to be observed in electron samples with even higher mobilities. However, there are no such reports in electron systems. The low field oscillations are more likely to be a consequence of magneto-intersubband scattering (MIS) due to the ZFSS, ${ }^{11-13}$ which also give rise to an additional $1 / B$-periodic term in the magneto-conductance as described above. Unlike the scattering between the electric subbands reported before, ${ }^{1-13}$ scattering happens between two spin subbands in our case.

One property of the MIS effect is that it is more insensitive to temperature than $\mathrm{SdH}$ oscillations. The MIS effect is temperature-independent because it depends on the alignments of Landau levels from two different subbands. The scattering possibility varies when two sets of Landau levels shifted relative to each other due to ZFSS (Fig. 1(c)). On the other hand, the SdH oscillations are more temperature dependent because it is related to the Fermi-Dirac distribution near the Fermi surface. Decreasing magnetic fields has a similar effect on the amplitude of $\mathrm{SdH}$ oscillations as increasing the temperature since the amplitude of $\mathrm{SdH}$ oscillations damps with the factor $Z / \sinh Z,{ }^{7,14}$ where $Z=2 \pi^{2} k_{B} T / \hbar \omega_{c}, T$ is the temperature, and $\omega_{c}$ is the cyclotron resonance frequency. In our samples, the low field oscillations keep robust at lower fields, whereas the $\mathrm{SdH}$ oscillations are severely damped (Fig. 1(b)).

In order to study the oscillation periods, we plot $\mathrm{i}$ versus $1 / \mathrm{B}_{\text {max }}$ for oscillation peaks in Fig. $2 ; \mathrm{i}=\Delta / \hbar \omega_{c}$ is an integer when the resistance reaches a local maximum and $\Delta$ is the gap of ZFSS. Despite the fact that the spin splitting in 2DHS is $k$-dependent, ${ }^{15}$ it is much more convenient to consider a simple scenario that the two spin subbands of heavy holes have a constant splitting energy $\Delta$, as illustrated in Fig. 1(c).

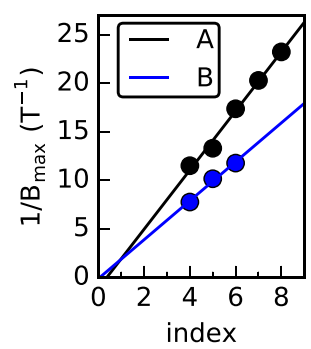

FIG. 2. The relation between oscillation indexes and reciprocal magnetic fields.

It is worth noting that the low field magnetoresistance in the longitudinal direction exhibits peaks at integer $i$ as the MIS oscillations of a two electric-subbands system does $\left[.{ }^{13}\right.$ The alignment of Landau levels in two subbands happens when $\Delta$ is an integer multiple of the cyclotron energy, thus giving a $1 / B$-periodic correction term in conductance with the period $\hbar \mathrm{e} / \mathrm{m}^{*} \Delta$, where e denotes the electron charge and $\mathrm{m}^{*}$ is the effective mass. The phase of the $1 / B$-periodic correction term is reported to have a difference of $\pi$ from the $\mathrm{SdH}$ term. In other words, the longitudinal conductance contributed by MIS encounters a maximum at an integer index, while the $\mathrm{SdH}$ shows a minimum. In the field region of interest, i.e., $|\mathrm{B}|>0.05 \mathrm{~T}, \rho_{x y}^{2} / \rho_{x x}^{2}>25 \gg 1$ for both samples; here, $\rho_{\mathrm{xy}}$ denotes the transverse resistivity, and $\rho_{\mathrm{xx}}$ denotes the longitudinal resistivity. Thus, the longitudinal conductivity $\sigma_{x x}=\rho_{x x} /\left(\rho_{x x}^{2}+\rho_{x y}^{2}\right) \approx \rho_{x x} / \rho_{x y}^{2} \propto \rho_{x x}$. The oscillation parts of $\rho_{x x}$ and $\sigma_{x x}$ have the same phase, which seems to be paradoxical at the first sight. The fitting lines are plotted in Fig. 2. The line from sample A intersects the index-axis near $(0.5,0)$ instead of $(0,0)$. This deviation from $(0,0)$ is probably due to the uncertainty in determining $1 / \mathrm{B}_{\max }$ at $\mathrm{i}=5$. The $1 / \mathrm{B}_{\max }$ in Fig. 2 is obtained by taking average over absolute values on both positive and negative fields.

The ZFSS gap $\Delta$ can be calculated with the formula

$$
\Delta=\hbar \omega_{\mathrm{c}, \mathrm{i}=1}=\hbar \mathrm{e} / \mathrm{m}^{*} \xi,
$$

where $\xi$ denotes the slope of the fitting line in Fig. 2. In order to determine the effective mass $\mathrm{m}^{*}$, we take $\mathrm{SdH}$ measurements at various temperatures in a $\mathrm{He} 3$ refrigerator. The effective masses are obtained by using a reduced version of Ando formula, ${ }^{7,14}$

$$
\ln (\mathrm{A} / \mathrm{T})=\mathrm{C}-\left(2 \pi^{2} \mathrm{k}_{B} \mathrm{~m}^{*} / \mathrm{e} \mathrm{B}\right) \mathrm{T},
$$

where $A$ is the amplitude of $\mathrm{SdH}$ oscillation and $C$ is a constant. The $\mathrm{SdH}$ oscillations and $\ln (\mathrm{A} / \mathrm{T})$ versus $T$ are illustrated in Fig. 3, where the effective masses of 0.54 and 0.44 (in unit of $\mathrm{m}_{e}$ ) are obtained for $\mathrm{A}$ and $\mathrm{B}$, respectively. Our mass result for sample $A$ is consistent with previous work on a double-sided doped $20 \mathrm{~nm}$ quantum well (QW) with a hole density of $1.1 \times 10^{11} \mathrm{~cm}^{-2}$ (Ref. 16) and on a double-sided doped $15 \mathrm{~nm}$ QW with $p \sim 1.94 \times 10^{11} \mathrm{~cm}^{-2}$. The smaller effective mass in sample B suggests a single side doped QW even though the sample is indeed symmetrically doped. An example of 2 DHS with $p=2.2 \times 10^{11} \mathrm{~cm}^{-2}$ in a single side doped $15 \mathrm{~nm}$ wide QW indicates an $m^{*}$ of 0.42 in Ref. 4 . The effective mass of holes in GaAs is sensitive to the confinement potential. In a single-sided doped QW, the asymmetric 


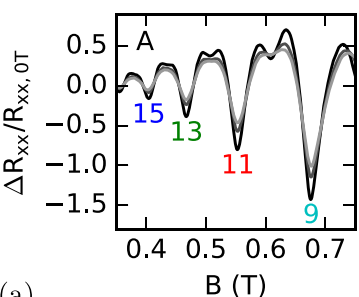

(a)

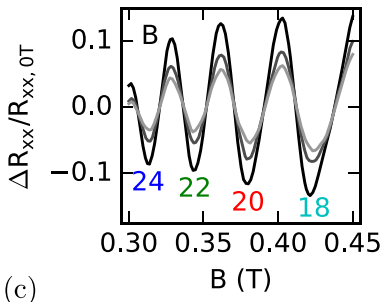

(b)
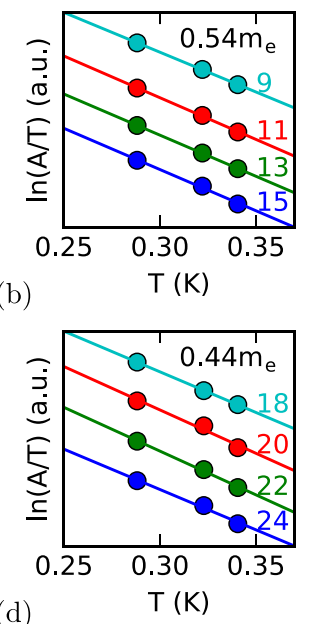

FIG. 3. The temperature-dependent SdH oscillations for sample A (panel (a)) and B (panel (c)). The magneto-resistances are normalized by the resistance at zero fields and are subtracted by a parabolic background within the range of (a) $0.2-1.2 \mathrm{~T}$ and (c) $0.3-0.45 \mathrm{~T}$. $\ln (A / T)$ vs $T$ is plotted to estimate the effective masses for sample (b) A and (d) B, with $A$ being the amplitudes of SdH oscillations and $T$ the temperatures. The lines in panel (d) are shifted for clarify. The colorful numbers indicate the filling factors.

doping decreases the effective width of confinement potential by introducing an internal electric field. As a result, the heavy hole mass will then become smaller as the gap between the heavy-hole ( $\mathrm{HH})$ band and the light-hole ( $\mathrm{LH})$ band is strengthened by decreasing QW width. ${ }^{16}$ The values of $\Delta$ are obtained by substituting the effective mass into formula (1). The results are listed in Table I.

When a voltage $V_{g}$ is applied to the front gate, peaks of low field oscillations in sample $\mathrm{C}$ are pushed towards lower fields as the density lowers, and vice versa, as shown in Figs. 4(a) and 4(d). The gate and the hole channel form a parallel plate capacitor; thus, the density displays a linear dependence on $V_{g}$, as shown in Fig. 4(c).

The shift of peak positions indicates the change in splitting gap $\Delta$. Since the electric field $E_{z}$ in growth direction provides a Rashba spin splitting proportional to itself, one would expect a symmetric evolution of $\Delta$ for positive and negative $V_{g}$ in a symmetrically doped QW wafer. However, this is not the case for sample C, as shown in Figs. 4(d) and 4(e). In order to extract the positions of low field maxima from the background signals in sample $\mathrm{C}$, we calculate the 2nd-order derivative of $R_{\mathrm{xx}}$ (Fig. 4(e)). The 2nd-order derivative is multiplied by $(-1)$ so that the maximum in $R_{\mathrm{xx}}$ remains a maximum after the data process. The relation of $B_{\mathrm{i}}$ vs. density is plotted in Fig. 4(f), with $B_{\mathrm{i}}$ the magnetic field at index $=i$ peak of $-\mathrm{dR}_{x x}^{2} / \mathrm{dB}^{2}$, taking average over absolute values at both positive and negative sides. $\Delta=\mathrm{B}_{\mathrm{i}=1}$ $=\mathrm{B}_{i} * \mathrm{i}$ is estimated by assuming a constant effective mass of 0.44 , which is from the results of sample B. A possible explanation for the asymmetric $\Delta$ versus gate voltage may come from the internal field, with different degrees of the ionization of two $\delta$-doping layers. This would also decreases the effective mass in the wafer as described above. Since the single-sided gate would change the charge distribution as well as the charge density, experiments with both front and back gates are necessary for further studies of the evolution of $\Delta$ under a tunable Rashba splitting.
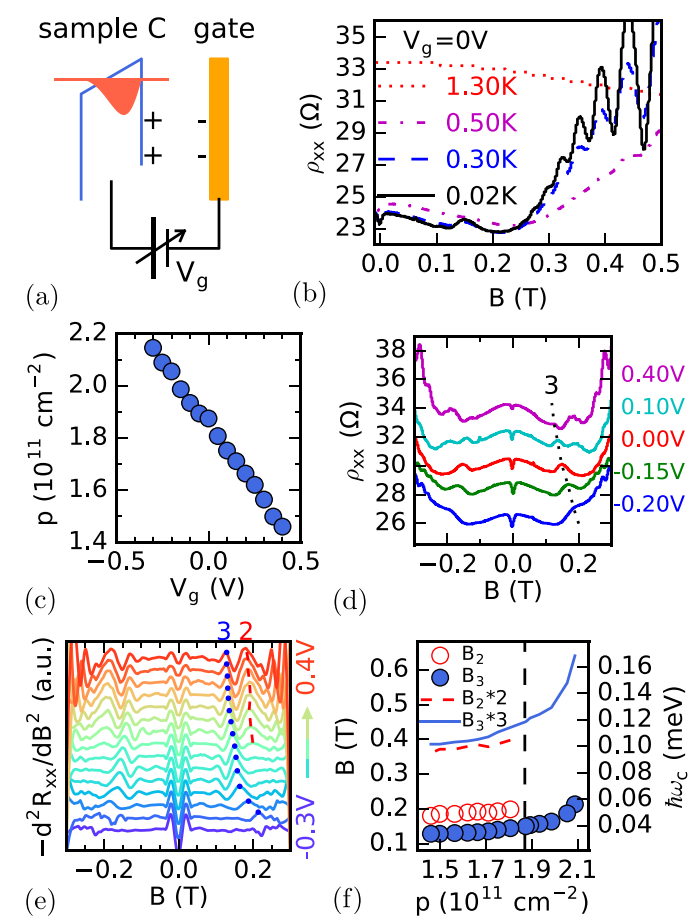

(d)
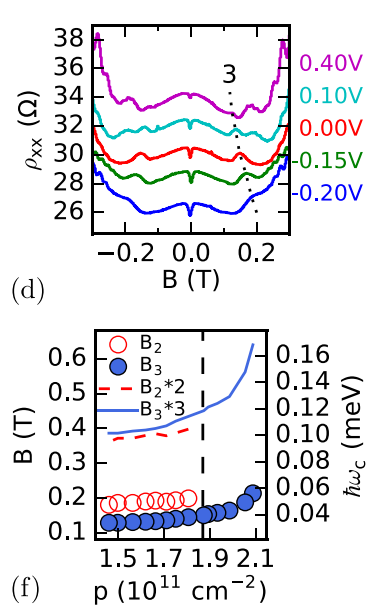

FIG. 4. The data from sample C. (a) The experimental setup for the gated device. A voltage $V_{g}$ is applied on the gate to tune the spin splitting and the density $p$. (b) The temperature-dependent low field oscillations smeared out at $500 \mathrm{mK}(\approx 0.04 \mathrm{meV})$. (c) Density vs. gate voltage $V_{g}$ shows a linear relation. (d) An overview of peak shifting under $V_{g}$. (e) The $V_{g}$-dependent low field oscillations. A 2nd-order derivative multiplied by $(-1)$ is used to subtract the background in order to highlight the oscillation phase. Each curve in (e) corresponds to a data point in (c). (f) The peak positions obtained from (e). The $B_{\text {index }}$ *index is shown as an estimation of spin splitting gap $\Delta$. The scale in energy is also labeled, assuming a constant effective mass of $0.44 m_{\mathrm{e}}$ taken from sample B. The dashed vertical line indicates the density at $V_{g}=0$.

The temperature-dependent low field oscillations in sample $\mathrm{C}$ are illustrated in Fig. 4(b). At base temperature, the oscillations are weaker than those in A and B because of the reduced mobility from sample fabrications.

In conclusion, we have reported low field magnetoresistance oscillations with zero field spin splitting. The low field oscillations arise from inter-spin-subband scattering and are $1 / B$ periodic with the period $\hbar \mathrm{e} / \Delta \mathrm{m}^{*}$. They show strong and weak amplitudes alternately at different indexes. The effect emerges before the onset of SdH oscillations. We use a front gate to tune the gap of ZFSS, which lead to a shift in the peaks of the low field oscillation. The gap of zero field spin splitting can be obtained by analyzing the oscillations, which is 0.07 and $0.13 \mathrm{meV}$ for samples $\mathrm{A}$ and $\mathrm{B}$, respectively. The observations of the low field oscillations benefit from the improved wafer quality and show potential to study spin related phenomena, such as spin Hall effect and resonant spin Hall effect. ${ }^{17,18}$

The work at Peking University was supported by National Basic Research Program of China grants 2012CB921301 and 2014CB920901, and by Collaborative Innovation Center of Quantum Matter. The work at Rice University was supported by NSF Grant (No. DMR-1508644) and Welch Foundation Grant (No. C-1682). The work at Princeton University was partially funded by the Gordon and 
Betty Moore Foundation as well as the National Science Foundation MRSEC Program through the Princeton Center for Complex Materials (No. DMR-0819860).

${ }^{1} \mathrm{R}$. Winkler, "Spin-orbit coupling effects in two-dimensional electron and hole systems," in Springer Tracts in Modern Physics (Springer-Verlag Berlin Heidelberg, 2003), Vol. 191.

${ }^{2}$ I. Zutic, J. Fabian, and S. Das Sarma, Rev. Mod. Phys. 76, 323 (2004).

${ }^{3}$ C. Gerl, S. Schmult, H.-P. Tranitz, C. Mitzkus, and W. Wegscheider, Appl. Phys. Lett. 86, 252105 (2005).

${ }^{4}$ Z. Q. Yuan, R. R. Du, M. J. Manfra, L. N. Pfeiffer, and K. W. West, Appl. Phys. Lett. 94, 052103 (2009).

${ }^{5}$ J. P. Lu, J. B. Yau, S. P. Shukla, M. Shayegan, L. Wissinger, U. Rössler, and R. Winkler, Phys. Rev. Lett. 81, 1282 (1998).

${ }^{6}$ S. J. Papadakis, E. P. De Poortere, H. C. Manoharan, M. Shayegan, and R. Winkler, Science 283, 2056 (1999).

${ }^{7}$ M. Hirmer, "High-mobility two-dimensional hole gases in III-V semiconductor heterostructures: Growth and transport properties," Ph.D. thesis, University of Regensburg, 2012.
${ }^{8}$ J. Luo, H. Munekata, F. F. Fang, and P. J. Stiles, Phys. Rev. B 38, 10142 (1988).

${ }^{9}$ F. Nichele, "Transport experiments in two-dimensional systems with strong spin-orbit interaction," Ph.D. thesis, ETH Zurich, 2014.

${ }^{10}$ J. Kunc, B. A. Piot, D. K. Maude, M. Potemski, R. Grill, C. Betthausen, D. Weiss, V. Kolkovsky, G. Karczewski, and T. Wojtowicz, Phys. Rev. B 92, 085304 (2015).

${ }^{11}$ M. E. Raikh and T. V. Shahbazyan, Phys. Rev. B 49, 5531 (1994).

${ }^{12}$ A. C. H. Rowe, J. Nehls, R. A. Stradling, and R. S. Ferguson, Phys. Rev. B 63, 201307 (2001).

${ }^{13}$ T. H. Sander, S. N. Holmes, J. J. Harris, D. K. Maude, and J. C. Portal, Phys. Rev. B 58, 13856 (1998).

${ }^{14}$ T. Ando, A. B. Fowler, and F. Stern, Rev. Mod. Phys. 54, 437 (1982).

${ }^{15}$ R. Winkler, Phys. Rev. B 62, 4245 (2000).

${ }^{16}$ H. Zhu, K. Lai, D. Tsui, S. Bayrakci, N. Ong, M. Manfra, L. Pfeiffer, and K. West, Solid State Commun. 141, 510 (2007).

${ }^{17}$ X.-J. Wu, T.-X. Li, C. Zhang, and R.-R. Du, Appl. Phys. Lett. 106, 012106 (2015).

${ }^{18}$ S.-Q. Shen, M. Ma, X. C. Xie, and F. C. Zhang, Phys. Rev. Lett. 92, 256603 (2004). 